\title{
Characteristic features of fungus ball in the maxillary sinus and the location of intralesional calcifications on computed tomographic images: A report of 2 cases
}

\author{
Jae-Hoon Lee $\mathbb{D}^{1}$, Byung-Do Lee $\mathbb{D}^{2, *}$ \\ ${ }^{1}$ Department of Otolaryngology, Institute of Wonkwang Medical Science, School of Medicine, Wonkwang University, Iksan, Korea \\ ${ }^{2}$ Department of Oral and Maxillofacial Radiology and Research Institute of Dental Education, College of Dentistry, Wonkwang University, \\ Iksan, Korea
}

\section{ABSTRACT}

This report presents 2 cases of sinus fungus ball and describes the characteristic radiographic features of fungus ball in the maxillary sinus. Two female patients, aged 62 and 40 years, sought consultations at a dental hospital for the treatment of dental implants and tooth pain, respectively. Panoramic radiography and small field-of-view (FOV) conebeam computed tomography (CBCT) did not provide detailed information for the radiographic diagnosis of fungus ball due to the limited images of the maxillary sinus. Additional paranasal sinus computed tomographic images showed the characteristic features of fungus ball, such as heterogeneous opacification and intralesional calcification of the maxillary sinus. The calcified materials of the fungus balls were located in the middle and superior regions of the maxillary sinus. It is necessary to use large-FOV CBCT for the detection of calcified materials in the upper maxillary sinus to confirm the diagnosis of fungus ball.(Imaging Sci Dent 2020; 50: 377-84)

KEY WORDS: Maxillary Sinus; Mycoses; Cone-Beam Computed Tomography

In 2009, the International Society for Human and Animal Mycology classified fungal rhinosinusitis into invasive and noninvasive forms based on histopathologic findings. ${ }^{1}$ The non-invasive type of fungal sinusitis mostly occurs in immunocompetent patients. However, certain invasive forms are associated with a high mortality rate and are common in immunocompromised patients. It has been proposed that a patient's immunocompromised state may predispose the patient to conversion to an invasive type. ${ }^{2}$ Fungus ball has been described as a non-invasive accumulation and dense conglomeration of fungal hyphae. ${ }^{1}$ Terms such as mycetoma, aspergilloma, and chronic noninvasive granuloma have been used for this entity. However, the term "fungus ball" is now favored. ${ }^{3}$ Fungus ball often affects a single sinus, usually the maxillary sinus, without the involvement of the mucosa, submucosa, bony structures,

This study was supported by Wonkwang University in 2019 .

Received July 13, 2020; Revised October 14, 2020; Accepted October 16, 2020 *Correspondence to : Prof. Byung-Do Lee

Department of Oral and Maxillofacial Radiology, College of Dentistry, Wonkwang University, Iksan-daero 460, Iksan, Jeonbuk 54538, Korea

Tel) 82-63-859-2912,E-mail) eebydo@wonkwang.ac.kr or blood vessels. ${ }^{4}$

It is important to differentiate fungus ball from chronic rhinosinusitis and other types of fungal sinusitis for proper treatment because invasive sinus disease may lead to death, and a misdiagnosis of fungus ball may lead to therapeutic misadventures. ${ }^{5,6}$

Among the currently available diagnostic tools, computed tomography (CT) is the optimal imaging technique for detecting fungus ball preoperatively. One of the most common CT findings is a partial or complete heterogeneous opacification of the involved sinus and the presence of intralesional hyperdensities with metal-like calcified materials. ${ }^{6,7}$ Patients with asymptomatic sinus fungus ball are occasionally diagnosed through the use of imaging studies that were performed for other purposes. If intralesional calcification of the maxillary sinus is detected, additional radiologic information should be obtained to confirm the diagnosis of fungus ball. Recently, cone-beam computed tomography (CBCT) has assumed a significant role in the diagnosis of disorders of the teeth, jaws, and sinuses. ${ }^{8,9}$ CBCT includes various fields of view (FOVs). ${ }^{10}$ Small- 


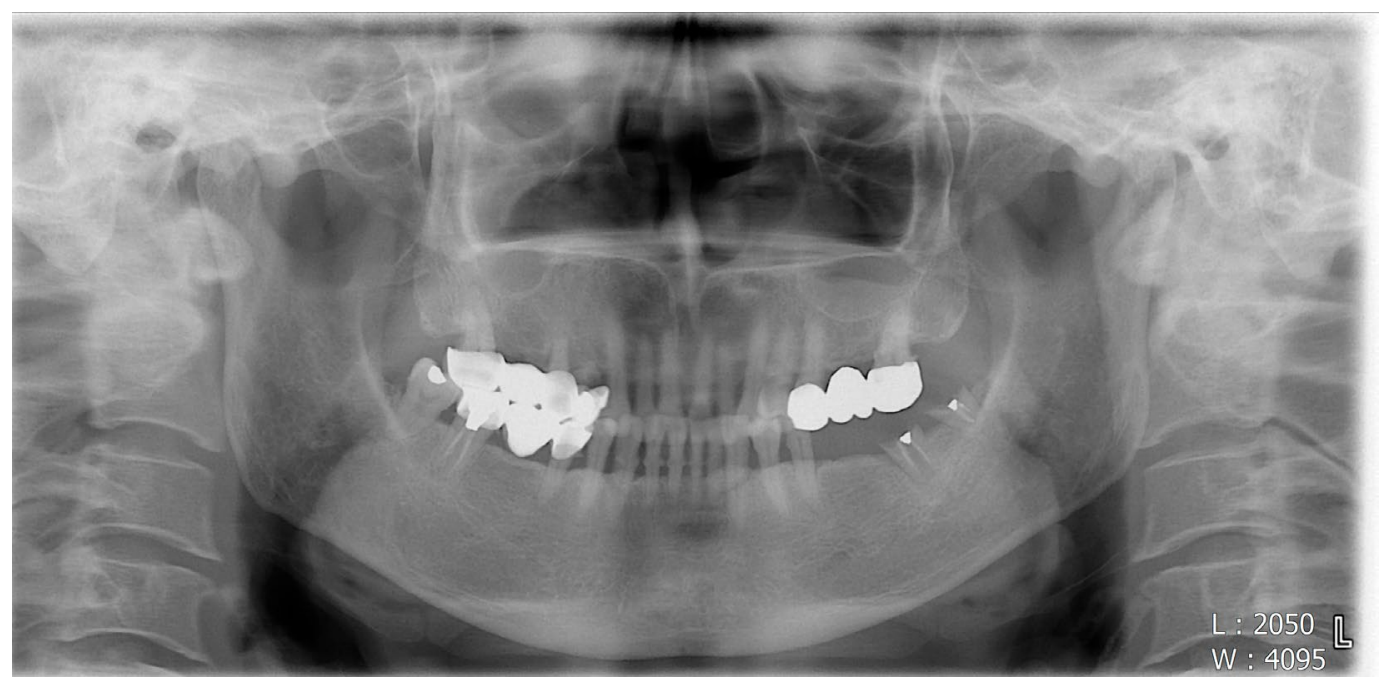

Fig. 1. Panoramic radiograph shows an edentulous region of the left posterior mandible and haziness of the right maxillary sinus, suggesting maxillary sinusitis.

FOV CBCT is usually used for dental problems due to its high resolution and low radiation dose. ${ }^{11}$ However, smallFOV CBCT may not show intralesional calcification in the upper region of the maxillary sinus; hence, a large-FOV CBCT is utilized in such cases due to its ability to detect calcifications in the upper maxillary sinus because it extends to the ostiomeatal complex and can cover the whole region of the maxillary sinus. It is necessary to select an optimal size of the FOV for CBCT to adequately detect calcifications anywhere in the maxillary sinus.

This report presents 2 cases of fungus ball with intralesional calcification in the middle and upper levels of the maxillary sinus. A literature review is also provided, with a particular focus on calcified materials in the maxillary sinus on CBCT.

\section{Case Report}

\section{Case 1}

A 62-year-old woman sought consultation at a dental hospital for a planned dental implant in the left mandibular first and second molars. A panoramic radiograph showed an edentulous region in the left posterior mandible and haziness of the right maxillary sinus (Fig. 1), suggesting maxillary sinusitis. However, CBCT covered only the mandible, the planned region for the dental implant. Detailed radiographic information on the right maxillary sinus was not obtained. Implantation of the left mandibular molar was performed, and prosthetic treatment was successful.

Four years after the implant therapy of the left mandible,

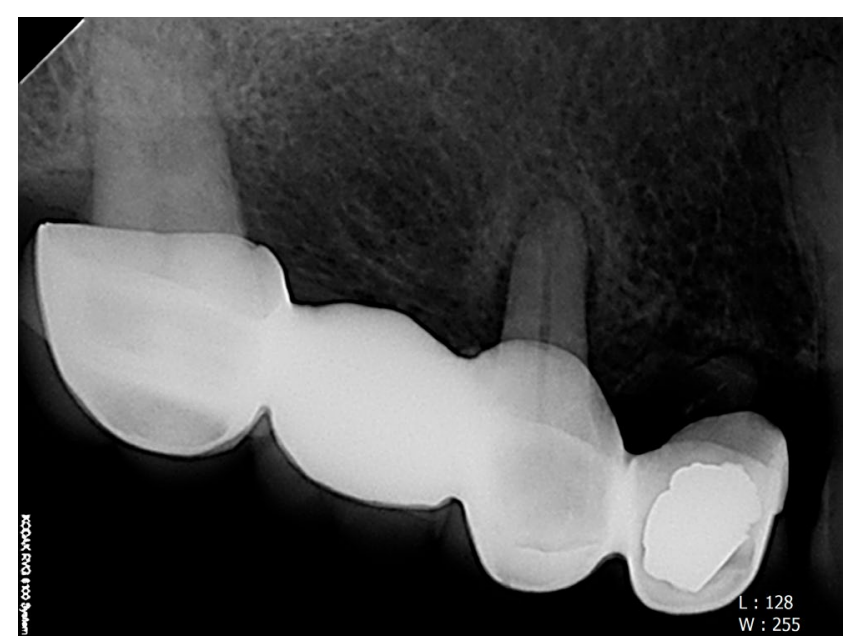

Fig. 2. Periapical radiograph shows severe alveolar bone loss of the right maxillary second premolar and second molar.

the right maxillary second premolar and second molar extracted due to severe tooth mobility (Fig. 2). The panoramic image still showed similar haziness in the right maxillary sinus (Fig. 3), implying a chronic and static inflammatory status. Medium-FOV CBCT $(169 \times 119 \mathrm{~mm}$, spatial resolution $0.2 \mathrm{~mm}$, Asahi Alphard Vega, Kyoto, Japan) showed a faint calcified material in the upper region of the right maxillary sinus (Fig. 4). A fungus ball was strongly suspected due to the intralesional calcification within the sinus opacification. The patient was recommended to receive paranasal sinus (PNS) CT (spatial resolution: 0.3 $\mathrm{mm}$, Somatom Definition Edge, Siemens, Erlangen, Germany) including the ostiomeatal complex. The PNS CT 


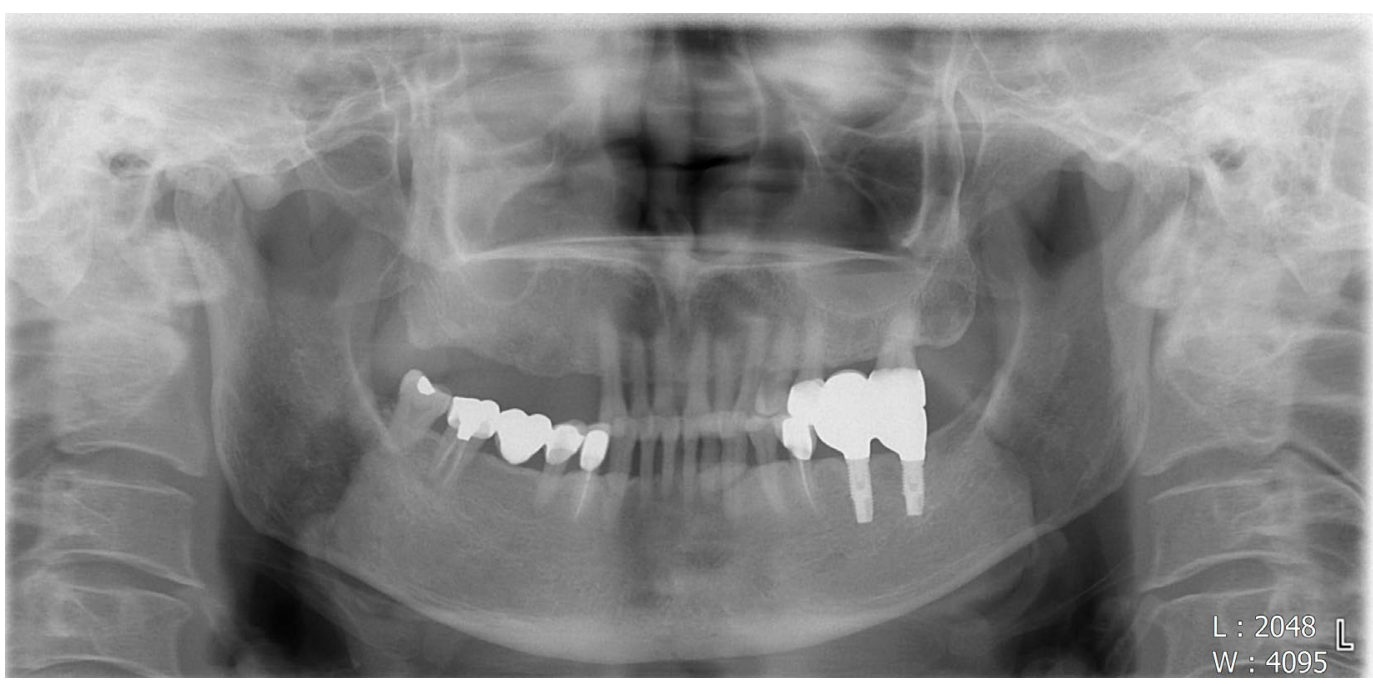

Fig. 3. Panoramic image shows similar haziness of the right maxillary sinus, compared with Figure 1.

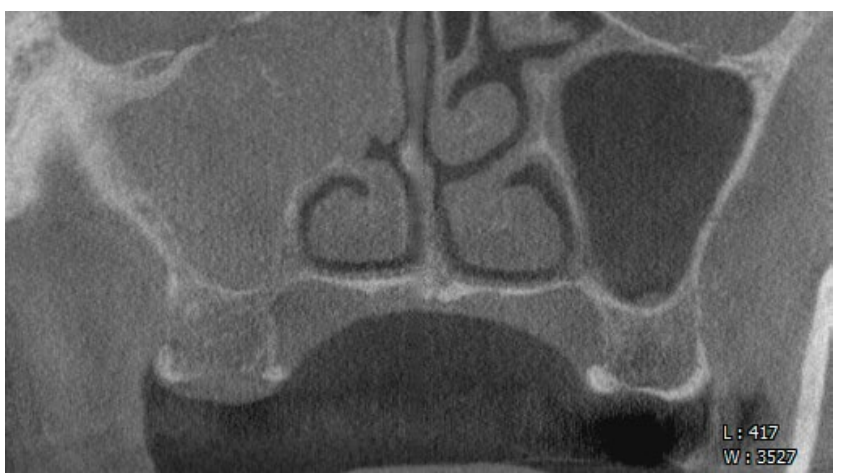

Fig. 4. Medium-sized coronal cone-beam computed tomographic image shows faint calcified material in the upper region of the right maxillary sinus.

showed heterogeneous, complete opacification with calcified materials in the right maxillary sinus on non-enhanced coronal images (Fig. 5A). Enhanced axial images presented sclerosis of the surrounding wall of the right maxillary sinus and nodular (Fig. 5B and C) and punctuate (Fig. 5C) calcified materials. These calcified materials presented in the middle and upper regions of the maxillary sinus. CT images also revealed sclerosis of the surrounding bony wall, bony erosion of the medial wall of the right maxillary sinus, and soft tissue protruding into the ostiomeatal complex (mass effect).

Endoscopic sinus surgery under general anesthesia revealed that the right maxillary sinus was filled with a brownish clay-like material, which was removed by saline irrigation and suctioning (Fig. 6). A histopathologic examination showed fungal hyphae with acute angle branching and septation (hematoxylin and eosin stain, $\times 400$, Fig. 7A). These hyphae were highlighted by the Gomori methenamine silver stain $(\times 400$, Fig. $7 B)$. These findings were morphologically consistent with Aspergillus infection. The patient remained disease-free during a 3-year follow-up period.

\section{Case 2}

A 40-year-old woman sought consultation at a dental hospital for the treatment of right upper first molar pain. The periapical view (Fig. 8) showed partial pulpal treatment status. She was in the process of receiving endodontic treatment at a private practice. Due to the suspicion of tooth fracture, CBCT with a small FOV $(102 \times 102$ $\mathrm{mm}$, spatial resolution: $0.1 \mathrm{~mm}$ ) was performed. A definite tooth fracture line in the right upper first molar was not seen. However, an opacification of the adjacent right maxillary sinus was partially and incidentally observed on parasagittal CBCT (Fig. 9). The primary working diagnosis was chronic maxillary sinusitis. Calcified materials in the upper region of the maxillary sinus were not observed because of the limited FOV of the CBCT, precluding the confirmation of the working diagnosis. Endodontic treatment was successfully completed. One year later, she visited the hospital's emergency room with severe pain around the right upper first molar. She also complained of nasal obstruction, right facial pain, and rhinorrhea. The PNS CT showed heterogeneous opacification, sclerosis of the surrounding bony wall, bony erosion of the medial wall of the right maxillary sinus, mass extension into the right nasal cavity, and mucosal thickening in the right ethmoid- 

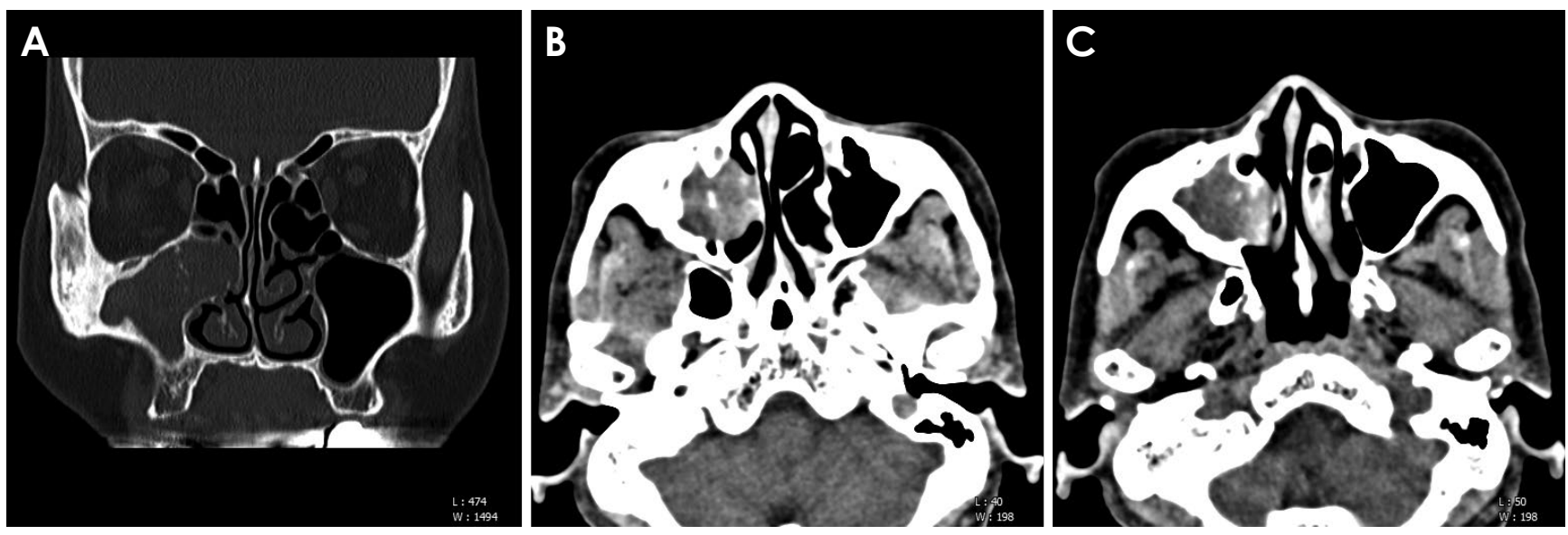

Fig. 5. A non-enhanced coronal computed tomographic image shows heterogeneous complete opacification with calcified materials in the central and upper region of the right maxillary sinus. These calcified materials show nodular (B and C) and punctate (C) configurations on the enhanced, consecutive axial images.

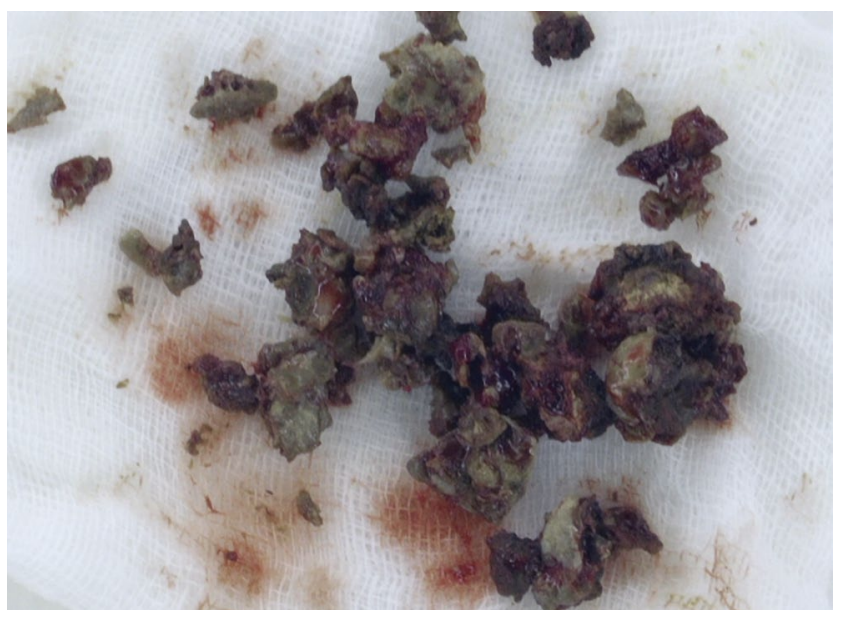

Fig. 6. Postoperative findings of endoscopic surgery shows brownish clay-like material in the right maxillary sinus.

al sinus (Fig. 10A). The CT image also showed calcified materials more distinctly on the enhanced CT image than on the non-enhanced CT image in the center and upper region of maxillary sinus on the coronal (Fig. 10B) and axial (Fig. 10C) images. These punctate and nodular calcifications were located in the middle and upper regions of the right maxillary sinus. The patient underwent endoscopic sinus surgery. Histopathologic findings showed a fungus ball with an intraluminal growth pattern and bundles of hyphae (Fig. 11). The patient remained disease-free during a 9-year follow-up period.

\section{Discussion}

Fungal sinusitis has been reported in about $10 \%$ of pati- ents requiring nose and sinus surgery, and between $13.5 \%$ and $28.5 \%$ of all maxillary sinusitis cases are of fungal or mixed fungal and bacterial etiologies. ${ }^{7,12}$ The non-invasive group of fungal sinusitis is composed of allergic fungal rhinosinusitis (AFRS) and fungus ball. The diagnostic criterion for AFRS is the presence of characteristic eosinophilrich, allergic mucin-containing fungal elements. However, eosinophilic mucin is not present in the sinonasal tract in patients with fungus ball. ${ }^{13}$ Fungus ball is the most common type of fungal disease and AFRS is extremely rare in Korea. ${ }^{14}$ To distinguish fungus ball from AFRS, the location of the disease is considered. AFRS is diffuse and bilateral and often associated with nasal polyps. The most commonly involved sinus in AFRS is the ethmoid sinuses. ${ }^{15}$ Conversely, fungus ball mostly occurs in isolation and unilaterally, and preferentially involves the maxillary sinus. ${ }^{7}$ Fungus ball is also frequently found in the sphenoid sinus, ${ }^{16}$ although frontal and ethmoidal fungus balls have been described. ${ }^{17}$ It has been reported that fungus ball may coexist with other forms of fungal sinusitis, including AFRS. ${ }^{6}$ Although AFRS is not considered invasive, if left untreated, the involved sinuses expand with subsequent intracranial or intraorbital extension, resulting in cranial or orbital symptoms. ${ }^{18}$

Fungus ball can be eradicated completely with proper surgical treatment. A prompt and accurate diagnosis of sinonasal fungus ball eliminates unnecessary medication and treatment delays. ${ }^{5}$ Fungus ball is more common in middle-aged and older women. ${ }^{19}$ Female predominance has been reported. Environmental and hormonal factors and the longer life expectancy of women may be a possible rationale for the female predominance. ${ }^{12,20}$ Fungus ball 

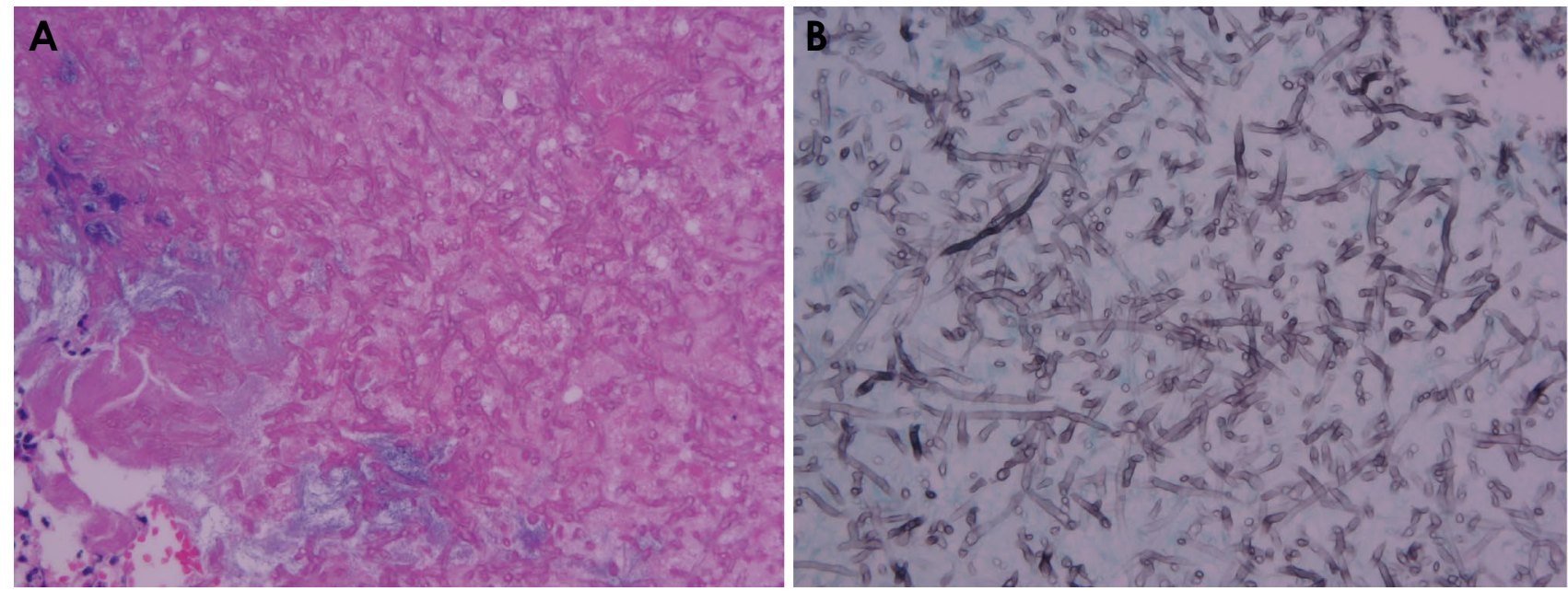

Fig. 7. A. Fungal hyphae with acute angle branching and septation are morphologically consistent with aspergillosis (hematoxylin and eosin stain, $\times 400)$. B. These hyphae are highlighted by Gomori methenamine silver stain $(\times 400)$.

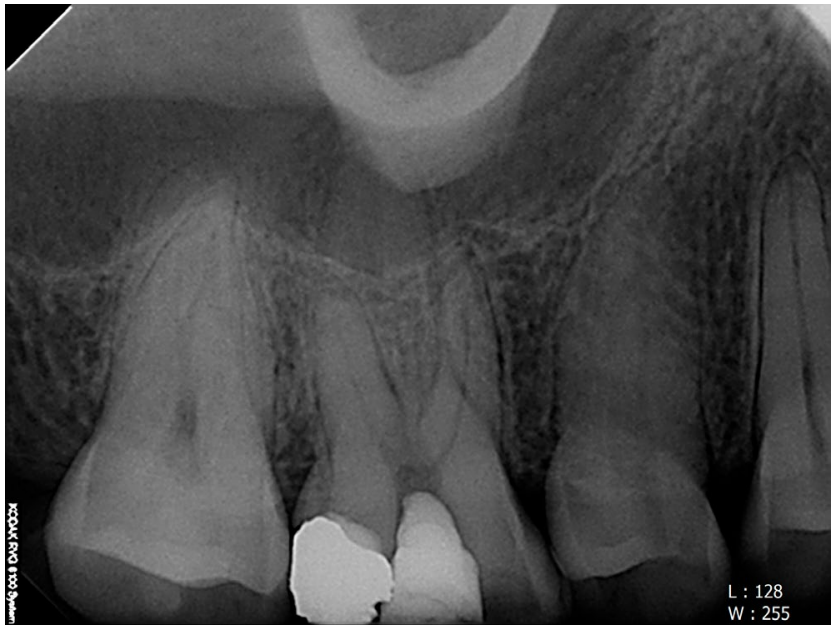

Fig. 8. Periapical radiograph shows partial pulpal treatment of the right maxillary first molar.

was once considered a relatively uncommon disease, but its incidence has increased dramatically over the last 2 decades. ${ }^{19}$ Although no definite reason for this increase is apparent, it is thought to be the result of improved knowledge of the disease, advances in diagnostic techniques, misuse of broad-spectrum antibiotics, and the aging population. ${ }^{7,20-22}$

The common CT findings of fungus ball are partial or often complete heterogeneous opacification of the involved sinus with intralesional calcifications and sclerosis of the bony wall of an opacified sinus. These features, although not pathognomonic, strongly suggest a fungus ball diagnosis. ${ }^{7}$ Fungus ball is more heterogeneous than other sinus opacities. ${ }^{23}$ Heterogeneous opacification of the involved sinus was observed in about $90 \%$ of partial or complete opacified

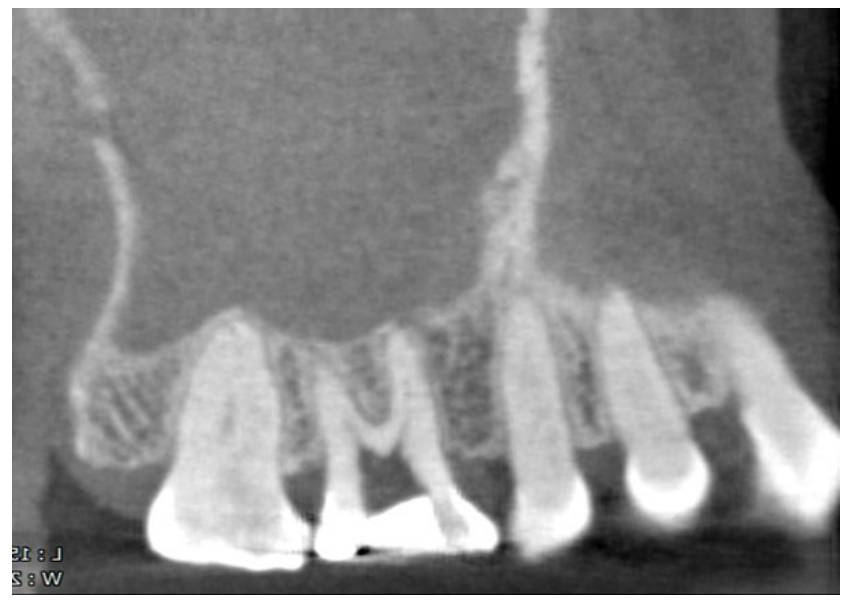

Fig. 9. A definite tooth fracture line is not seen, but almost full opacification of the right maxillary sinus is incidentally, but partially, observed on the parasagittal cone-beam computed tomographic image.

sinuses, whereas homogenous opacification was observed in $10 \%$ of cases. ${ }^{7}$ Sinus fungus ball typically appears as a hyperattenuation on non-contrast CT scans because of the dense fungal hyphae and metabolic deposits. These matted fungal hyphae and metabolic deposits, as a conglomerate solid mass in the sinus, ${ }^{5}$ may contribute to the high-density opacities in the sinus fungus ball. The detection of metallic hyperdensity on a CT scan suggests the presence of metal metabolites from the fungal organism. It has been reported that calcium phosphate and calcium sulfate were present within the fungus ball, especially in the necrotic center of the fungal mass corresponding to areas of high attenuation. ${ }^{7,24}$ Several heavy metallic elements, including copper, 

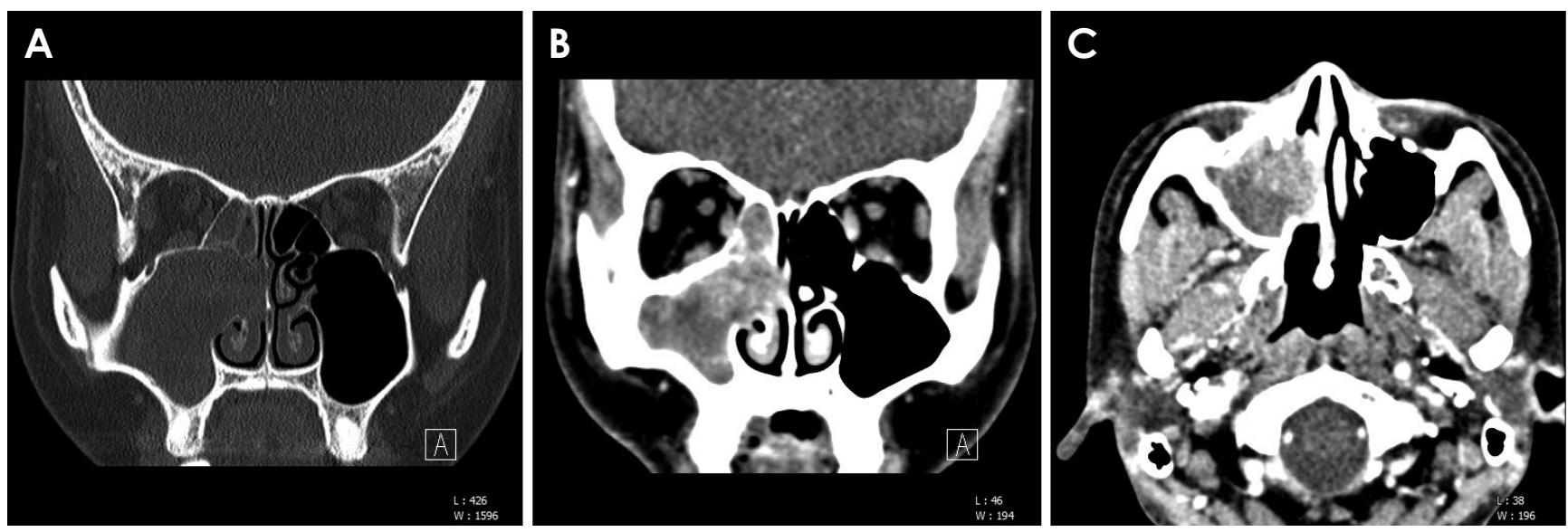

Fig. 10. A. Bony erosion of the medial wall of the right maxillary sinus, mass extension into right nasal cavity, and mucosal thickening in the right ethmoidal sinus are seen on non-enhanced computed tomography (CT). Enhanced CT shows calcified materials more distinctly than non-enhanced $\mathrm{CT}$ in the center and upper region of maxillary sinus on coronal(B) and axial views (C).
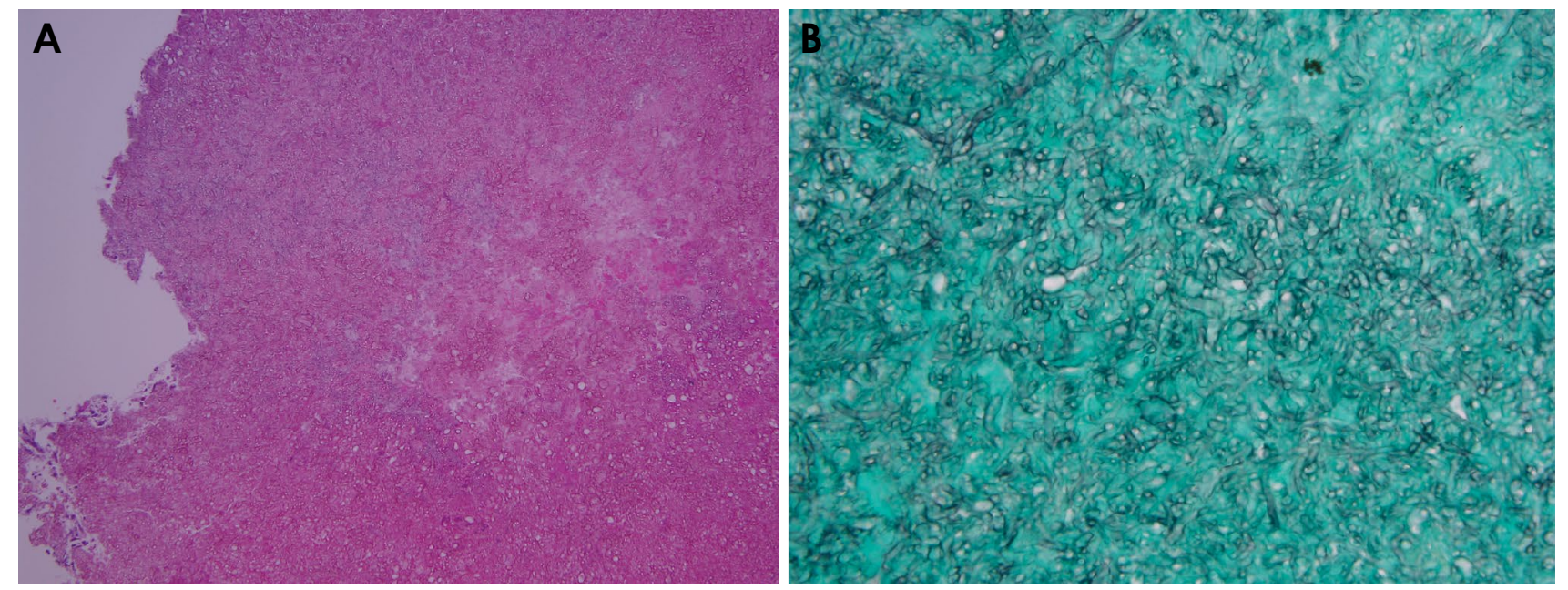

Fig. 11. A. Histopathologic examinations show a fungus ball with an intraluminal growth pattern. No tissue invasion is seen (hematoxylin and eosin stain, $\times 100$ ), B. At high power, the fungus ball consists of tight bundles of hyphae (Gomori methenamine-silver stain, $\times 400$ ).

zinc, iron, and calcium, are essential for fungal growth and metabolic activation. As metallic elements, zinc and calcium also contribute to micro-calcifications or spots with a metallic density on the CT image. ${ }^{25}$ There are various ranges in prevalence rate of intrasinus calcification on CT scans in patients with fungus ball which has been reported to be $88 \%,{ }^{26} 77 \%,{ }^{27}$ and on the other $50 \%-83 \%{ }^{28}$. The sensitivity and specificity of CT imaging, using sinus opacification and the presence of areas with hyperattenuation as a diagnostic criterion for fungus ball, were reported to be $62 \%$ and $99 \%$, respectively. If intralesional calcification spots are not present on CT images of the sinuses, it is difficult to diagnose fungus ball in the maxillary sinus preoperatively. ${ }^{29}$

Cha et $a .^{28}$ reported that the sensitivity of only one CT feature of intralesional hyperdensity in fungus ball was rather low (0.749), and there was a considerable risk of false negative results. They concluded that it is necessary to add clinical features of age and other radiographic features, such as full haziness with the mass effect, to increase the sensitivity of CT for the diagnosis of fungus ball in the maxillary sinus. The mass effect was defined as widening of the ostiomeatal complex or thinning of the maxillary sinus wall. It has been reported that if inadequate mucociliary clearance of the fungi occurs, these fungi produce an inflammatory response from the sinus mucosa, which soon becomes too large to escape the natural ostium of the sinus. This process continues until the sinus is full and can then become secondarily bacterially infected or expanded. ${ }^{15}$ 
In this report, complete opacification and soft tissue protrusion into the ostiomeatal complex were noted in both cases. These features are compatible with the aforementioned mass effect. ${ }^{28}$ It is not easy to distinguish sinonasal fungus ball from other forms of fungal or unilateral sinusitis based on radiologic findings alone, because the intralesional densities may also be found in AFRS and/or nonfungal maxillary sinusitis. ${ }^{5,16,28}$ The location of the calcifications on sinus CT was also categorized as peripheral, central, or mixed pattern according to its location within the sinus CT scan. ${ }^{5}$ Most of the calcifications in fungal sinusitis were found to be centrally located within the maxillary sinus, while calcifications in nonfungal sinusitis were usually found at the periphery, near the wall of the maxillary sinus. The central location of the calcification can be explained by the fact that the calcification develops within the mycelial mass, which is usually located in the center of the maxillary sinus. ${ }^{16}$ Nodular or linear-shaped calcifications were found in both fungal and nonfungal sinusitis; however, fine punctate calcification was found only in fungal sinusitis, while round or eggshell-type calcifications were found exclusively in the nonfungal variety. ${ }^{5,27}$ A flocculent type of fungus ball was also reported. ${ }^{6}$ The PNS CT in this report sufficiently showed the detailed images of the calcified materials. However, calcified materials with sizes smaller than $0.3 \mathrm{~mm}$ would not be visualized by PNS CT. The window level of CT may be altered to enhance the contrast between the focal high-density area and the surrounding inflammatory tissue for the optimal evaluation of fine calcifications. ${ }^{5}$ Jiang et al. ${ }^{25}$ assumed that the sensitivity could be increased by improving the resolution of CT images. Although the spatial resolution of CBCT in this study, which was approximately $0.1-0.2 \mathrm{~mm}$, was superior to that of PNS CT, CBCT could not provide detailed information on the calcified materials due to the limited FOV and low contrast resolution. Punctate or nodular, intralesional calcified materials were clearly detected on PNS CT, but were not visible in the CBCT findings of case 2 (small FOV, $0.1 \mathrm{~mm}$ ) and faintly seen in the CBCT findings of case 1 (medium FOV, $0.2 \mathrm{~mm}$ ). These calcified materials were located in the upper and central regions of the maxillary sinus in both cases beyond the FOV of CBCT in this report. The spatial resolution of CBCT in this report was sufficient, but FOV was not adequate for the detection of intralesional calcifications and mass effect in the ostiomeatal unit.

CBCT is often the first sectional imaging technique used for workup of maxillary sinus disease because it is now widely available in the dental field due to its relatively low radiation dose and high spatial resolution. Identification of an area of increased attenuation in maxillary sinus on CBCT can direct the dentist to focus on calcified materials. This enables the confirmation of fungus ball that would be otherwise be diagnosed as mucosal thickening or maxillary sinusitis. It is necessary to optimize the scan size of CBCT for the detection of upper sinus calcification.

\section{Conflicts of Interest: None}

\section{References}

1. Chakrabarti A, Denning DW, Ferguson BJ, Ponikau J, Buzina W, Kita H, et al. Fungal rhinosinusitis: a categorization and definitional schema addressing current controversies. Laryngoscope 2009; 119: 1809-18.

2. Ferguson BJ. Fungus balls of the paranasal sinuses. Otolaryngol Clin North Am 2000; 33: 389-98.

3. Yoon YH, Xu J, Park SK, Heo JH, Kim YM, Rha KS. A retrospective analysis of 538 sinonasal fungus ball cases treated at a single tertiary medical center in Korea (1996-2015). Int Forum Allergy Rhinol 2017; 7: 1070-5.

4. Huang Z, Xu H, Xiao N, Li Y, Dong Y, Li Y, et al. Predictive significance of radiographic density of sinus opacity and bone thickness in unilateral maxillary sinus mycetoma. ORL J Otorhinolaryngol Relat Spec 2019; 81: 111-20.

5. Seo YJ, Kim J, Kim K, Lee JG, Kim CH, Yoon JH. Radiologic characteristics of sinonasal fungus ball: an analysis of 119 cases. Acta Radiol 2011; 52: 790-5.

6. deShazo RD, O’Brien M, Chapin K, Soto-Aguilar M, Swain R, Lyons $\mathrm{M}$, et al. Criteria for the diagnosis of sinus mycetoma. $\mathbf{J}$ Allergy Clin Immunol 1997; 99: 475-85.

7. Grosjean P, Weber R. Fungus balls of the paranasal sinuses: a review. Eur Arch Otorhinolaryngol 2007; 264: 461-70.

8. Shokri A, Faradmal MJ, Hekmat B. Correlations between anatomical variations of the nasal cavity and ethmoidal sinuses on cone-beam computed tomography scans. Imaging Sci Dent 2019; 49: 103-13.

9. Vestin Fredriksson M, Öhman A, Flygare L, Tano K. When maxillary sinusitis does not heal: findings on CBCT scans of the sinuses with a particular focus on the occurrence of odontogenic causes of maxillary sinusitis. Laryngoscope Investig Otolaryngol 2017; 2: 442-6.

10. Dillenseger JP, Gros CI, Sayeh A, Rasamimanana J, Lawniczak F, Leminor JM, et al. Image quality evaluation of small FOV and large FOV CBCT devices for oral and maxillofacial radiology. Dentomaxillofac Radiol 2017; 46: 20160285.

11. Tang L, Zhou XD, Wang Y, Zhang L, Zheng QH, Huang DM. Detection of vertical root fracture using cone beam computed tomography: report of two cases. Dent Traumatol 2011; 27: 484-8.

12. Nicolai P, Lombardi D, Tomenzoli D, Villaret AB, Piccioni M, Mensi M, et al. Fungus ball of the paranasal sinuses: experience in 160 patients treated with endoscopic surgery. Laryngoscope 2009; 119: 2275-9.

13. Tsai TL, Guo YC, Ho CY, Lin CZ. The role of ostiomeatal com- 
plex obstruction in maxillary fungus ball. Otolaryngol Head Neck Surg 2006; 134: 494-8.

14. Han DH, An SY, Kim SW, Kim DY, Rhee CS, Lee CH, et al. Primary and secondary fungal infections of the paranasal sinuses: clinical features and treatment outcomes. Acta Otolaryngol 2007; 127 Suppl 558: 78-82.

15. Ni Mhurchu E, Ospina J, Janjua AS, Shewchuk JR, Vertinsky AT. Fungal rhinosinusitis: a radiological review with intraoperative correlation. Can Assoc Radiol J 2017; 68: 178-86.

16. Yoon JH, Na DG, Byun HS, Koh YH, Chung SK, Dong HJ. Calcification in chronic maxillary sinusitis: comparison of CT findings with histopathologic results. AJNR Am J Neuroradiol 1999; 20: 571-4.

17. Braun JJ, Bourjat P. CT imaging of fungal and nonfungal caseous sinusitis. A report of 50 cases. J Radiol 2000; 81: 227-31.

18. Aribandi M, McCoy VA, Bazan C III. Imaging features of invasive and noninvasive fungal sinusitis: a review. Radiographics 2007; 27: 1283-96.

19. Dufour X, Kauffmann-Lacroix C, Ferrie JC, Goujon JM, Rodier MH, Karkas A, et al. Paranasal sinus fungus ball and surgery: a review of 175 cases. Rhinology 2005; 43: 34-9.

20. Dufour X, Kauffmann-Lacroix C, Ferrie JC, Goujon JM, Rodier MH, Klossek JM. Paranasal sinus fungus ball: epidemiology, clinical features and diagnosis. A retrospective analysis of 173 cases from a single medical center in France, 1989-2002. Med Mycol 2006; 44: 61-7.

21. Kim JS, So SS, Kwon SH. The increasing incidence of paranasal sinus fungus ball: a retrospective cohort study in two hundred forty-five patients for fifteen years. Clin Otolaryngol 2017;
42: 175-9.

22. Lim HS, Yoon YH, Xu J, Kim YM, Rha KS. Isolated sphenoid sinus fungus ball: a retrospective study conducted at a tertiary care referral center in Korea. Eur Arch Otorhinolaryngol 2017; 274: 2453-9.

23. Killeen DE, Sedaghat AR, Cunnane ME, Gray ST. Objective radiographic density measurements of sinus opacities are not strong predictors of noninvasive fungal disease. Am J Rhinol Allergy 2014; 28: 483-6.

24. Stammberger H, Jakse R, Beaufort F. Aspergillosis of the paranasal sinuses $\mathrm{x}$-ray diagnosis, histopathology, and clinical aspects. Ann Otol Rhinol Laryngol 1984; 93: 251-6.

25. Jiang Z, Zhang K, Huang W, Yuan Q. A preliminary study on sinus fungus ball with microCT and X-ray fluorescence technique. PLoS One 2016; 11: e0148515.

26. Zinreich SJ, Kennedy DW, Malat J, Curtin HD, Epstein JI, Huff LC, et al. Fungal sinusitis: diagnosis with CT and MR imaging. Radiology 1988; 169: 439-44.

27. Chang T, Teng MM, Wang SF, Li WY, Cheng CC, Lirng JF. Aspergillosis of the paranasal sinuses. Neuroradiology 1992; 34: 520-3.

28. Cha H, Song Y, Bae YJ, Won TB, Kim JW, Cho SW, et al. Clinical characteristics other than intralesional hyperdensity may increase the preoperative diagnostic accuracy of maxillary sinus fungal ball. Clin Exp Otorhinolaryngol 2020; 13: 157-63.

29. Dhong HJ, Jung JY, Park JH. Diagnostic accuracy in sinus fungus balls: CT scan and operative findings. Am J Rhinol 2000; 14: $227-31$. 